\title{
Bipartite Diametrical Graphs of Diameter 4 and Extreme Orders
}

\author{
Salah Al-Addasi ${ }^{1}$ and Hasan Al-Ezeh ${ }^{2}$ \\ ${ }^{1}$ Mathematics Department, Faculty of Science, Hashemite University, Zarqa 150459, Jordan \\ ${ }^{2}$ Mathematics Department, Faculty of Science, University of Jordan, Amman 11942, Jordan \\ Correspondence should be addressed to Salah Al-Addasi, salah@hu.edu.jo
}

Received 9 July 2007; Accepted 5 December 2007

Recommended by Peter Johnson

\begin{abstract}
We provide a process to extend any bipartite diametrical graph of diameter 4 to an $S$-graph of the same diameter and partite sets. For a bipartite diametrical graph of diameter 4 and partite sets $U$ and $W$, where $2 m=|U| \leq|W|$, we prove that $2^{m}$ is a sharp upper bound of $|W|$ and construct an $S$-graph $G\left(2 m, 2^{m}\right)$ in which this upper bound is attained, this graph can be viewed as a generalization of the Rhombic Dodecahedron. Then we show that for any $m \geq 2$, the graph $G\left(2 m, 2^{m}\right)$ is the unique (up to isomorphism) bipartite diametrical graph of diameter 4 and partite sets of cardinalities $2 m$ and $2^{m}$, and hence in particular, for $m=3$, the graph $G(6,8)$ which is just the Rhombic Dodecahedron is the unique (up to isomorphism) bipartite diametrical graph of such a diameter and cardinalities of partite sets. Thus we complete a characterization of $S$-graphs of diameter 4 and cardinality of the smaller partite set not exceeding 6 . We prove that the neighborhoods of vertices of the larger partite set of $G\left(2 m, 2^{m}\right)$ form a matroid whose basis graph is the hypercube $Q_{m}$. We prove that any $S$-graph of diameter 4 is bipartite self complementary, thus in particular $G\left(2 m, 2^{m}\right)$. Finally, we study some additional properties of $G\left(2 m, 2^{m}\right)$ concerning the order of its automorphism group, girth, domination number, and when being Eulerian.
\end{abstract}

Copyright (C) 2008 S. Al-Addasi and H. Al-Ezeh. This is an open access article distributed under the Creative Commons Attribution License, which permits unrestricted use, distribution, and reproduction in any medium, provided the original work is properly cited.

\section{Introduction}

There are frequent occasions in which graphs with a lot of symmetries are required. One such family of graphs is that of $S$-graphs. $S$-graphs form a special type of symmetric diametrical graphs which are one of the most interesting classes of diametrical graphs, see [1,2], as well as of $k$-pairable graphs introduced in [3]. Diametrical graphs were studied under different names, see $[2,4-6]$.

All graphs we consider are assumed to be finite, connected, and to have no loops or multiple edges. For undefined notions and terminology, we use [7].

Two vertices, $u$ and $v$, of a nontrivial connected graph $G$ are said to be diametrical if $d(u, v)=\operatorname{diam}(G)$. A nontrivial connected graph $G$ is called diametrical if each vertex $v$ of 
$G$ has a unique diametrical vertex $\bar{v}$, the vertex $\bar{v}$ is called the buddy of $v$, see [4] and [5]. A diametrical graph $G$ is called minimal if removing any edge of $G$ produces a graph which is not diametrical of the same diameter. A diametrical graph $G$ in which $\bar{u} \bar{v} \in E(G)$, whenever $u v \in E(G)$, is called harmonic. A diametrical graph $G$ is called maximal if there is no diametrical graph $H$ with the same vertex set and diameter as $G$ such that $E(H)$ contains $E(G)$ properly. A diametrical graph $G$ is called symmetric if $d(u, v)+d(v, \bar{u})=\operatorname{diam}(G)$ for all $u, v \in V(G)$, see [2], that is, a diametrical graph $G$ is symmetric if and only if $V(G)=I(u, \bar{u})$ for any $u \in V(G)$, where $I(u, \bar{u})$ denotes the interval of $G$ consisting of all vertices on shortest $(u, \bar{u})$-paths, see [5]. A symmetric diametrical graph is called an antipodal graph, and a bipartite antipodal graph is called an $S$-graph, see [1].

It is shown in [8] that the concepts of symmetric and maximal are equivalent in the class of bipartite diametrical graphs of diameter 4 . We provide a process to extend any given bipartite diametrical graph of diameter 4 to a maximal diametrical graph and hence an $S$-graph of the same diameter and partite sets.

For a bipartite diametrical graph of diameter 4 and partite sets $U$ and $W$ with $|U| \leq|W|$, it is given in [8] that $|W| \leq\left(\begin{array}{c}|U| \\ (1 / 2)|U|\end{array}\right)$. In this paper, we improve this upper bound by proving that $|W| \leq 2^{m}$, where $m=(1 / 2)|U|$. To show that $2^{m}$ is a sharp upper bound, we construct a bipartite diametrical graph, which we denote by $G\left(2 m, 2^{m}\right)$, of diameter 4 in which this maximum value of $|W|$ is attained. In particular, when $m=3$, the graph $G\left(2 m, 2^{m}\right)$ is indeed just the Rhombic Dodecahedron which is sometimes also called the Rhomboidal Dodecahedron, see [9]. The Rhombic Dodecahedron appears in some applications in natural sciences, see, for example, $[10,11]$. It is the convex polyhedron depicted in Figure 1 and can be built up by placing six cubes on the faces of a seventh, taking the centers of outer cubes and the vertices of the inner as its vertices, and joining the center of each outer cube to the closer four vertices of the inner. The number $|U|$ is a sharp lower bound of $|W|$ which is previously known through a graph constructed in [8], which we denote in this paper by $G(2 m, 2 m)$. For $m=3$, it is proven in [12] that, up to isomorphism, the graph $G(2 m, 2 m)=K_{2} \times C_{6}$ is the unique symmetric diametrical graph of order 12 and diameter 4 . For larger $m, G(2 m, 2 m)$ needs not be the unique symmetric diametrical bipartite graph of diameter 4 . We give an example of an $S$-graph with the same cardinalities of partite sets as those of $G(8,8)$ but not isomorphic to $G(8,8)$. On the other hand, we prove that, for any $m \geq 2$, the graph $G\left(2 m, 2^{m}\right)$ is not only the unique (up to isomorphism) $S$-graph of diameter 4 and partite sets of cardinalities $2 m$ and $2^{m}$, but also is the unique bipartite diametrical graph of such a diameter and partite sets. Consequently, the Rhombic Dodecahedron is the unique diametrical graph of diameter 4 and maximum order where the smaller partite set has cardinality 6. Joining this to the main result of [12], our result about the upper bound of the cardinality of the larger partite set completes a characterization of $S$-graphs of diameter 4 and cardinality of the smaller partite set not exceeding 6.

Matroids constitute an important unifying structure which has many equivalent definitions. According to one of these definitions, a matroid on a finite set $I$ is a collection $\beta$ of subsets of $I$, called bases, which satisfies the exchange property: for all $A, B \in \beta$ and $a \in A-B$, there exists $b \in B-A$ such that $(A-\{a\}) \cup\{b\} \in \beta$. It is well known that all the bases of a matroid have the same cardinality. The basis graph $G(\beta)$ of a matroid $\beta$ is the graph whose vertices are the bases of the matroid, where two vertices $A, B$ are adjacent if they differ by a single exchange, that is, if the symmetric difference $(A-B) \cup(B-A)$ has exactly two elements. The basis graphs faithfully represent their matroids, see [13] and references therein; thus studying the basis graph amounts to studying the matroid itself. We prove that the neighborhoods of 


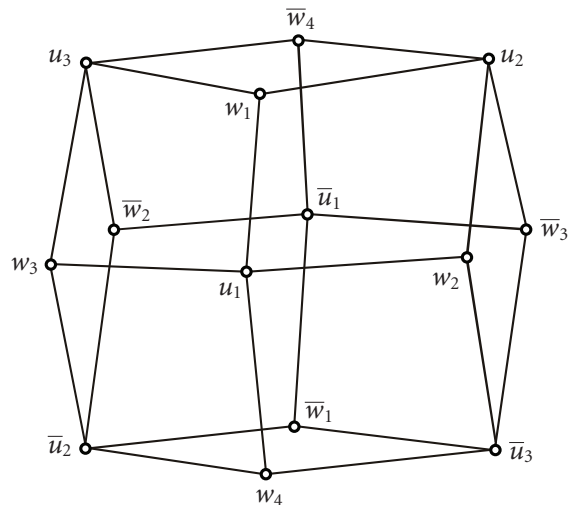

Figure 1: The Rhombic Dodecahedron.

the vertices of the larger partite set of $G\left(2 m, 2^{m}\right)$ form a matroid whose basis graph is the hypercube $Q_{m}$. The hypercubes together with the Rhombic Dodecahedra appear as the cells in the tight span of a totally split-decomposable metric which is used in the field of phylogenetic analysis, see [14].

A bipartite graph $G$ with partite sets $U$ and $W$ is called bipartite self complementary if $G$ is a subgraph of $K_{|U|,|W|}$ which is isomorphic to its complement $K_{|U|,|W|}-E(G)$ with respect to the complete bipartite graph with the same partite sets, see [15]. We show that the graphs $G\left(2 m, 2^{m}\right)$ and $G(2 m, 2 m)$ are bipartite self complementary and study some of their properties concerning girth, domination number, order of automorphism group, and when being Eulerian.

\section{A sharp upper bound of the cardinality of the larger partite set}

One basic property of any bipartite diametrical graph of even diameter is that each of the partite sets has an even number of elements, as stated in Lemma 2.1, see [8].

Lemma 2.1. If $G$ is a bipartite diametrical graph of even diameter $d$ and partite sets $U$ and $W$, then for each $v \in V$, both $v$ and $\bar{v}$ belong to the same partite set, and hence $|U|$ and $|W|$ are even.

In particular, when $\operatorname{diam}(G)=4$, each partite set of $G$ consists of a vertex $v$, its buddy $\bar{v}$, and those vertices equidistant to $v$ and $\bar{v}$.

It is shown in [2] that symmetric diametrical graphs form a proper special class of maximal diametrical graphs. But the two concepts coincide in the class of bipartite diametrical graphs of diameter 4 according to the following fact which was proven in [8].

Lemma 2.2. Let $\mathrm{G}$ be a bipartite diametrical graph of diameter 4 . Then the following are equivalent:

(i) $G$ is maximal in the class of bipartite diametrical graphs with the same partite sets as $G$;

(ii) $G$ is maximal;

(iii) $G$ is symmetric.

The following theorem includes a process to extend any given bipartite diametrical graph of diameter 4 to a bipartite diametrical graph of the same diameter and partite sets, 
which is maximal in the class of bipartite diametrical graphs with the same partite sets, and hence, by the previous lemma, is an $S$-graph.

Theorem 2.3. Let $G$ be a bipartite diametrical graph of diameter 4 and partite sets $U$ and $W$. Then, the following hold:

(i) if $u \in U$ and $w \in W$ such that the set $\{u, \bar{u}, w, \bar{w}\}$ of vertices is independent, then the graph $G+u w$ is bipartite diametrical of the same diameter and partite sets as $G$;

(ii) if $u w \in E(G)$ but $\bar{u} \bar{w} \notin E(G)$, then the graph $G+\bar{u} \bar{w}$ is bipartite diametrical of the same diameter and partite sets as $G$;

(iii) if $H$ is a graph obtained from $G$ by repeated applications of (i) and (ii) until none of them is applicable, then $H$ is an S-graph of the same diameter and partite sets as $G$.

Proof. (i) Assume to the contrary that $G+u w$ is either not diametrical or has diameter less than 4 . Then there exists a vertex $v \in V(G)$ such that $d_{G+u w}(v, \bar{v})<4$. But, since $G+u w$ is also bipartite with the same partite sets as $G$ and both $v, \bar{v}$ belong to the same part of $V(G)$, then $d_{G+u w}(v, \bar{v})$ is even. Hence $d_{G+u w}(v, \bar{v})=2$. Thus there exists a $(v, \bar{v})$-path $P$ in $G+u w$ containing the new edge $u w$ and having length 2 . Then either $\{v, \bar{v}\}=\{u, \bar{u}\}$ and $P$ is $u w \bar{u}$ or $\{v, \bar{v}\}=\{w, \bar{w}\}$ and $P$ is $\bar{w} u w$, which gives a contradiction in both cases since $w \bar{u}, \bar{w} u \notin E(G+$ $u w)$. Therefore, $G+u w$ is a diametrical graph of diameter 4 .

(ii) Let $u w \in E(G)$ such that $\bar{u} \bar{w} \notin E(G)$ with $u \in U$ and $w \in W$, where $U$ and $W$ are the partite sets of $G$. Then $\bar{u} w \notin E(G)$ because otherwise $d_{G}(u, \bar{u})=2$, which is not the case. Similarly, $u \bar{w} \notin E(G)$. Now, we will show by contradiction that $G+\bar{u} \bar{w}$ is diametrical of diameter 4. Assume not, then there exists a vertex $v \in V(G)$ such that $d_{G+\bar{u} \bar{w}}(v, \bar{v})<4$. Clearly, $G+\bar{u} \bar{w}$ is bipartite with the same partite sets as $G$ since $\bar{u} \in U$ and $\bar{w} \in W$. But both $v$ and $\bar{v}$ belong to the same part of $V(G)$, hence $d_{G+\bar{u} \bar{w}}(v, \bar{v})$ is even, and so $d_{G+\bar{u} \bar{w}}(v, \bar{v})=2$. Thus, there exists a $(v, \bar{v})$-path $P$ in $G+\bar{u} \bar{w}$ containing the new edge $\bar{u} \bar{w}$ and having length 2 . Consequently, either $\{v, \bar{v}\}=\{u, \bar{u}\}$ and $P$ is $\bar{u} \bar{w} u$ or $\{v, \bar{v}\}=\{w, \bar{w}\}$ and $P$ is $w \bar{u} \bar{w}$, which gives a contradiction in both cases since $u \bar{w}, \bar{u} w \notin E(G+\bar{u} \bar{w})$. Therefore, $G+\bar{u} \bar{w}$ is bipartite diametrical of diameter 4 .

(iii) Let $H$ be a graph obtained from $G$ by addition of edges according to (i) or (ii) until none of them can be applied. By Lemma 2.2, it would be enough to show that $H$ is maximal in the class of bipartite diametrical graphs of diameter 4 and partite sets $U$ and $W$. Assume to the contrary that there exist two nonadjacent vertices $u \in U$ and $w \in W$ of $H$ such that $H+u w$ still diametrical of diameter 4. Then, since (ii) is no more applicable on $H$, we have $\bar{u} \bar{w} \notin E(H)$. But also (i) is not applicable on $H$, so the set $\{u, \bar{u}, w, \bar{w}\}$ is not independent, therefore either $u \bar{w} \in E(H)$ or $\bar{u} w \in E(H)$. Consequently, either $d_{H}(w, \bar{w})=2$ or $d_{H}(u, \bar{u})=2$, a contradiction.

The only bipartite diametrical graph of diameter 4 in which the smaller partite set has cardinality less than 6 is the 8-cycle as Lemma 2.4 (proven in [8]) says.

Lemma 2.4. If $G$ is a bipartite diametrical graph of diameter 4 and partite sets $U$ and $W$ with $|U| \leq$ $|W|$, then $|U| \geq 6$ unless $G=C_{8}$.

For a bipartite diametrical graph of diameter 4 and partite sets $U$ and $W$ with $|U| \leq|W|$, we have $|U| \neq 2$, and whenever $|U|=4$, we must have $|W|=4$. It was shown in [8] that if $|U| \geq 6$, then $|W| \leq\left(\begin{array}{c}2 m \\ m\end{array}\right)$, where $m=(1 / 2)|U|$. Thus for $|U|=6$, we have $|W| \leq 20$. This is not a sharp upper bound, as shown at the end of this section. In order to obtain a sharp upper bound, we start by the following lemma. 
Lemma 2.5. Let $u$ and $v$ be distinct vertices of a diametrical graph $G$ of diameter $d \geq 3$. Then $N(u) \nsubseteq N(v)$.

Proof. If $u=\bar{v}$, then $N(u) \cap N(v)=\phi$ since $d(u, v) \geq 3$. Suppose that $u \neq \bar{v}$ and assume to the contrary that $N(u) \subseteq N(v)$. Any shortest path joining $\bar{v}$ and $u$ must include a vertex from $N(u)$; let $x$ be such a vertex in such a path. Then $d(\bar{v}, u)=d(\bar{v}, x)+d(x, u)=d(\bar{v}, x)+1$. But $x \in N(u) \subseteq N(v)$ and $v$ is the only vertex of $G$ at distance $d$ from $\bar{v}$, hence $d(\bar{v}, x)=d-1$. Therefore, $d(\bar{v}, u)=(d-1)+1=d$, contradicting the uniqueness of the buddy vertex of $\bar{v}$.

In particular, two distinct vertices in a diametrical graph of diameter at least 3 cannot have the same neighborhood.

Before tending to give an upper bound of $|W|$, let us recall the following fact proven in [8].

Lemma 2.6. Every vertex $v$ of an $S$-graph $G$ of diameter 4 is adjacent to exactly half of the vertices in the partite set of $G$ not containing $v$.

We are now in a position to prove our improvement of the upper bound of $|W|$.

Theorem 2.7. Let $G$ be a bipartite diametrical graph of diameter 4 and partite sets $U$ and $W$, where $2 m=|U| \leq|W|$. Then, $|W| \leq 2^{m}$.

Proof. By Theorem 2.3, there exists an $S$-graph $H$ with the same diameter and partite sets as $G$ which has $G$ as a subgraph. Then, by Lemma 2.6 , each vertex $w \in W$ has exactly $m$ neighbors in $U$. Clearly, $N_{H}(w)$ cannot contain a vertex and its buddy. But Lemma 2.5 implies that the $|W|$ sets $N_{H}(w), w \in W$ are mutually distinct. Hence, $|W|$ must be less than or equal to the number of ways of choosing single representatives from each of $m$ two-element sets; clearly that number is $2^{m}$.

Now, if $G$ is a bipartite diametrical graph of diameter 4 and partite sets $U$ and $W$, where $|U| \leq|W|$, then by Lemma $2.1,|U|=2 m$ for some positive integer $m$. Since $\operatorname{diam}(G)=4$, we must have $m \geq 2$. In the next section, we will construct for each integer $m \geq 2$ a bipartite diametrical graph of diameter 4 and partite sets $U$ and $W$ with $|U|=2 m$ and $|W|=2^{m}$, which is the maximum possible cardinality of $W$ according to the previous theorem. Consequently, the upper bound of $|W|$ given in Theorem 2.7 is indeed sharp.

\section{Constructing the graph $G\left(2 m, 2^{m}\right)$ with maximum cardinality of the larger partite set}

For a bipartite diametrical graph of diameter 4 and partite sets $U$ and $W$ with $2 m=|U| \leq|W|$, an obvious lower bound of $|W|$ is $2 m$. An $S$-graph of diameter 4 and partite sets with equal cardinalities was constructed in [8] as follows.

Lemma 3.1. For any integer $m \geq 2$, the graph $G$ whose vertex set is the disjoint union $U \cup W$, where $U=\left\{u_{1}, \bar{u}_{1}, u_{2}, \bar{u}_{2}, \ldots, u_{m}, \bar{u}_{m}\right\}$ and $W=\left\{w_{1}, \bar{w}_{1}, w_{2}, \bar{w}_{2}, \ldots, w_{m}, \bar{w}_{m}\right\}$, and edge set is

$$
\begin{aligned}
E(G) & =\left\{u_{i} w_{t}: 1 \leq i \leq m, 1 \leq t \leq m+1-i\right\} \\
& \cup\left\{u_{i} \bar{w}_{t}: 1 \leq i \leq m, m+1-i<t \leq m\right\} \\
& \cup\left\{\bar{u}_{i} \bar{w}_{t}: 1 \leq i \leq m, 1 \leq t \leq m+1-i\right\} \\
& \cup\left\{\bar{u}_{i} w_{t}: 1 \leq i \leq m, m+1-i<t \leq m\right\},
\end{aligned}
$$

is an S-graph of diameter 4. 


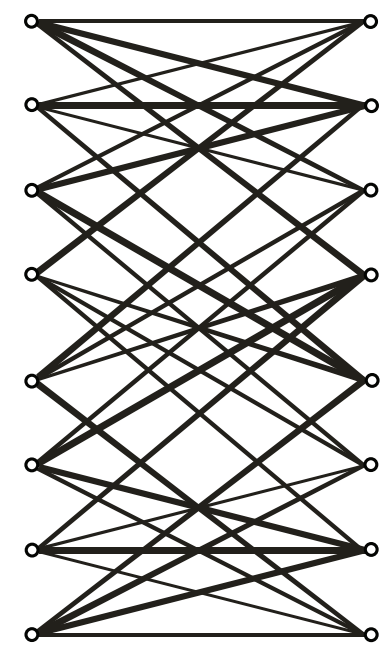

$G(8,8)$

(a)

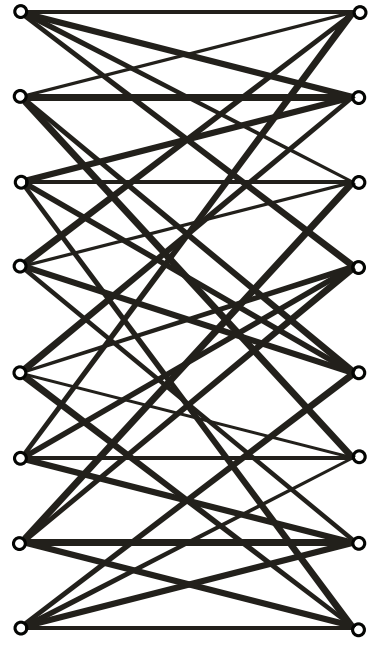

$H$

(b)

Figure 2: Two nonisomorphic $S$-graphs with minimum $|W|$. Any two vertices of $H$ at distance 2 from each other have exactly 2 common neighbors.

We will denote the graph $G$ in Lemma 3.1 by $G(2 m, 2 m)$. The graph $G(8,8)$ is depicted in Figure 2(a).

The next theorem provides an example of a bipartite diametrical graph of diameter 4 and partite sets $U$ and $W$ with $|U| \leq|W|$ in which the maximum possible value of $|W|$ given in Theorem 2.7 is attained.

Theorem 3.2. Suppose that $m>1$ is an integer. There is a unique S-graph of diameter 4 with partite sets $U, W$ satisfying $|U|=2 m$ and $|W|=2^{m}$.

Proof. Let $U=\left\{u_{1}, u_{1}^{*}, \ldots, u_{m}, u_{m}^{*}\right\}$ and $W=\left\{w_{1}, \ldots, w_{2^{m}}\right\}$. By Lemma 2.6 and the proof of Theorem 2.7 there is only one way to make an $S$-graph of diameter 4 with partite sets $U, W$ and with $u_{i}$ and $u_{i}^{*}$ being buddies, $i=1, \ldots, m$ : put the $w_{j}$ into one-to-one correspondence with the $2^{m}$ different sets $S=\left\{u_{1}^{\prime}, \ldots, u_{m}^{\prime}\right\}$ in which $u_{i}^{\prime}$ is one of $u_{i}, u_{i}^{*}$ for each $i=1, \ldots, m$, and define adjacency by setting $N\left(w_{j}\right)=S$ if $w_{j}$ corresponds to $S$ in the one-to-one correspondence. It is straightforward to see that the graph so defined is an $S$-graph of diameter 4; for each $w$ in $W$, the buddy of $w$ is the vertex in $W$ whose set of neighbors is $U-N(w)$.

We will denote the graph constructed in the proof of Theorem 3.2 by $G\left(2 m, 2^{m}\right)$.

The following fact follows immediately from the constructions of the graphs $G(2 m, 2 m)$ and $G\left(2 m, 2^{m}\right)$.

Corollary 3.3. For any integer $m \geq 2$, if $G$ is a bipartite diametrical graph of diameter 4 and partite sets $U$ and $W$, where $2 m=|U| \leq|W|$, then $2 m \leq|W| \leq 2^{m}$, where both inequalities are sharp.

Example 3.4. For $m=3$, the graph $G\left(2 m, 2^{m}\right)=G(6,8)$ which we have already constructed is indeed the Rhombic Dodecahedron depicted in Figure 1. 


\section{Some properties of the graphs $G(2 m, 2 m)$ and $G\left(2 m, 2^{m}\right)$}

We have seen in the previous section that for any integer $m \geq 2$ and partite sets $U$ and $W$ with $2 m=|U| \leq|W|$, the graph $G(2 m, 2 m)$ is a bipartite diametrical graph of diameter 4 and such partite sets with minimum possible order, while $G\left(2 m, 2^{m}\right)$ is one with maximum possible order.

Now we will show that the graph $G(2 m, 2 m)$ is always included within the graph $G\left(2 m, 2^{m}\right)$, recall that a subgraph $H$ of $G$ is isometric if $d_{G}(u, v)=d_{H}(u, v)$ for all $u, v \in V(H)$, see [16].

Theorem 4.1. For any integer $m \geq 2$, the graph $G\left(2 m, 2^{m}\right)$ has an isometric subgraph isomorphic to $G(2 m, 2 m)$.

Proof. Let $U=\left\{u_{1}, \bar{u}_{1}, u_{2}, \bar{u}_{2}, \ldots, u_{m}, \bar{u}_{m}\right\}$ and $W=\left\{w_{1}, w_{2}, \ldots, w_{2^{m}}\right\}$ be the partite sets of the graph $G\left(2 m, 2^{m}\right)$. Then, by the construction of $G\left(2 m, 2^{m}\right)$, for each $i=1,2, \ldots, m$, there exists a unique vertex $y_{i} \in W$ such that $N\left(y_{i}\right)=\left\{u_{i}: 1 \leq i \leq m+1-i\right\} \cup\left\{\bar{u}_{i}: m+1-i<i \leq\right.$ $m\}$, and hence $N\left(\bar{y}_{i}\right)=\left\{\bar{u}_{i}: 1 \leq i \leq m+1-i\right\} \cup\left\{u_{i}: m+1-i<i \leq m\right\}$. Then,the set of vertices $U \cup\left\{y_{1}, \bar{y}_{1}, y_{2}, \bar{y}_{2}, \ldots, y_{m}, \bar{y}_{m}\right\}$ induces a subgraph $H$ of $G\left(2 m, 2^{m}\right)$ isomorphic to $G(2 m, 2 m)$. Obviously, the buddy of a vertex of $H$ in $H$ is precisely its buddy in $G\left(2 m, 2^{m}\right)$. To show that $H$ is isometric, let $x$ and $z$ be any two distinct vertices of $H$. If $x=\bar{z}$ or $x z \in E(H)$, then $d_{H}(x, z)=d_{G\left(2 m, 2^{m}\right)}(x, z)$. So suppose that $x \neq \bar{z}$ and $x z \notin E(H)$. Then it is clear that $d_{H}(x, z)=d_{G\left(2 m, 2^{m}\right)}(x, z)=2$ when both $x$ and $z$ belong to the same partite set of $H$. Thus let $x$ and $z$ be in different partite sets of $H$, say $x \in U$ and $z \in W_{H}=\left\{y_{1}, \bar{y}_{1}, y_{2}, \bar{y}_{2}, \ldots, y_{m}, \bar{y}_{m}\right\}$. Then $x z \notin E\left(G\left(2 m, 2^{m}\right)\right)$ for otherwise we would have $N_{G\left(2 m, 2^{m}\right)}(z) \supseteq N_{H}(z) \cup\{x\}$ which contradicts the fact that $\operatorname{deg}_{G\left(2 m, 2^{m}\right)} z=m$. Then, $d_{H}(x, z)=d_{G\left(2 m, 2^{m}\right)}(x, z)=3$. Therefore $H$ is an isometric subgraph of $G\left(2 m, 2^{m}\right)$.

The Rhombic Dodecahedron and the even cycles $C_{2 n}(n \geq 2)$ are minimal diametrical graphs, while the graph $K_{2} \times C_{6}$ is not, for if we remove an edge $e$ corresponding to $K_{2}$, then the obtained graph $\left(K_{2} \times C_{6}\right)-e$ is again diametrical of the same diameter. Notice also that the graph $K_{2} \times C_{6}$ is just $G(6,6)$, which means that $G(2 m, 2 m)$ needs not be minimal. But the graph $G\left(2 m, 2^{m}\right)$ is minimal diametrical as the next result says.

Theorem 4.2. For any integer $m \geq 2$, if $H$ is an $S$-graph of diameter 4 and partite sets of cardinalities $2 m$ and $2^{m}$, then $H$ is a minimal diametrical graph.

Proof. By Theorem 3.2, the graph $H$ is isomorphic to $G\left(2 m, 2^{m}\right)$. Let $u w$ be an edge of $G=G\left(2 m, 2^{m}\right)$, where $u \in U$ and $w \in W$. Then $N_{G-u w}(w)=N_{G}(w)-\{u\}, N_{G}(\bar{w})=$ $U-N_{G}(w)$, and $\bar{u} \in N_{G}(\bar{w})$. Let $x \in W$ be the vertex whose neighborhood in $G$ is $N_{G}(x)=\left(N_{G}(\bar{w})-\{\bar{u}\}\right) \cup\{u\}$. Then $N_{G-u w}(x) \cap N_{G-u w}(w)=\phi$. Hence, $d_{G-u w}(w, x) \neq 2$. But $d_{G-u w}(w, x)$ is even, so $d_{G-u w}(w, x) \geq 4$. Clearly $x \neq \bar{w}$ since $u \notin N_{G}(\bar{w})$. Therefore, $x$ and $\bar{w}$ are two distinct vertices with $d_{G-u w}(w, x) \geq 4$ and $d_{G-u w}(w, \bar{w}) \geq 4$, which implies that $G-u w$ is not diametrical of diameter 4 . Since $u w$ was an arbitrary edge of $G\left(2 m, 2^{m}\right)$, the graph $H$ which is isomorphic to $G\left(2 m, 2^{m}\right)$ is minimal.

Now we can improve Theorem 3.2 and show that the graph $G\left(2 m, 2^{m}\right)$ is not only the unique (up to isomorphism) $S$-graph of diameter 4 and partite sets of cardinalities $2 m$ and $2^{m}$, but also the unique (up to isomorphism) bipartite diametrical graph of such a diameter and partite sets. 
Theorem 4.3. For any integer $m \geq 2$, if $G$ is a bipartite diametrical graph of diameter 4 and partite sets of cardinalities $2 m$ and $2^{m}$, then $G$ is isomorphic to $G\left(2 m, 2^{m}\right)$.

Proof. Let $G$ be a bipartite diametrical graph of diameter 4 and partite sets $U_{G}$ and $W_{G}$ with $\left|U_{G}\right|=2 m$ and $\left|W_{G}\right|=2^{m}$. In view of Theorem 3.2, it would be enough to show that $G$ is symmetric. So, assume to the contrary that $G$ is not symmetric. Then, by Theorem 2.3, the graph $G$ is a proper subgraph of an $S$-graph $H$ of the same diameter and partite sets as $G$. Let $u w \in E(H)-E(G)$. Then $G \subseteq H-u w \subseteq H$, and hence for any $x, y \in V(G)$, we have $d_{G}(x, y) \geq d_{H-u w}(x, y) \geq d_{H}(x, y)$. Therefore, $H-u w$ is diametrical of the same diameter and partite sets as $G$ and $H$, which contradicts the minimality of $H$ implied by the previous theorem.

The following characterization of the Rhombic Dodecahedron $G(6,8)$ is a direct consequence of Theorem 4.3 and Theorem 2.7 .

Corollary 4.4. The Rhombic Dodecahedron is the unique (up to isomorphism) bipartite diametrical graph of diameter 4 and partite sets $U$ and $W$, where $6=|U|<|W|$.

According to [12], if $G$ is an $S$-graph of order $n$ and diameter 4 with partite sets $U$ and $W$ such that $G \neq C_{8}$, then $n \geq 12$. Moreover, if $n=12$, then $|U|=|W|=6$. It is also shown in [12] that $K_{2} \times C_{6}$ is the unique (up to isomorphism) symmetric diametrical graph of order 12 and diameter 4 and hence in particular the unique $S$-graph of such an order and diameter. The previous corollary shows that the Rhombic Dodecahedron is not only the unique (up to isomorphism) S-graph of order 14 and diameter 4, but also the unique (up to isomorphism) bipartite diametrical graph of such an order and diameter. Combining these results with Lemma 2.4, we can completely characterize the $S$-graphs of diameter 4 and cardinality of the smaller partite set not exceeding 6 .

Corollary 4.5. Let $G$ be an S-graph of diameter 4 and cardinality of the smaller partite set not exceeding 6. Then $G$ is isomorphic to one of the graphs: $C_{8}, K_{2} \times C_{6}$ and the Rhombic Dodecahedron.

In the set of bipartite diametrical graphs of diameter 4 and partite sets $U$ and $W$ with $2 m=|U| \leq|W|$, although the graph $G\left(2 m, 2^{m}\right)$ is the unique (up to isomorphism) with maximum order, the graph $G(2 m, 2 m)$ needs not be the only one with minimum order, for example, the graphs $K_{2} \times C_{6}=G(6,6)$ and $\left(K_{2} \times C_{6}\right)-e$, where $e$ is an edge correspoding to $K_{2}$, both have minimum order. Even under the condition of being symmetric, although $G(6,6)$ was proven in [12] to be the only one, $G(2 m, 2 m)$ in general needs not be unique, for example, $G(8,8)$ and the graph $H$ of diameter 4 in Figure 2 are nonisomorphic $S$-graphs with minimum order.

Now we will prove that the set of all neighborhoods of vertices of the larger partite set in the graph $G\left(2 m, 2^{m}\right)$ forms a matroid on the smaller partite set, and determine its basis graph.

Theorem 4.6. Let $U$ and $W$ be the partite sets of $G\left(2 m, 2^{m}\right)$ with cardinalities $2 m$ and $2^{m}$, respectively. Then $\beta=\{N(w): w \in W\}$ forms a matroid on $U$ whose basis graph is the hypercube $Q_{m}$.

Proof. Let $N\left(w_{i}\right), N\left(w_{j}\right)$ be two elements of $\beta$ with $a \in N\left(w_{i}\right)-N\left(w_{j}\right)$. Then $\bar{a} \in N\left(w_{j}\right)$. Thus the set $S=\left(N\left(w_{i}\right)-\{a\}\right) \cup\{\bar{a}\}$ has exactly $m$ elements and contains no pair of diametrical vertices. So by the construction of $G\left(2 m, 2^{m}\right)$, there exists a vertex $w_{k} \in W$ such that $N\left(w_{k}\right)=S$. Therefore, $\left(N\left(w_{i}\right)-\{a\}\right) \cup\{\bar{a}\} \in \beta$ and hence $\beta$ is a matroid. Now to determine the basis graph of $\beta$, let $N\left(w_{j}\right)$ be a base of the matroid $\beta$ obtained from 
a base $N\left(w_{i}\right)$ by a single exchange. Then $N\left(w_{j}\right)=\left(N\left(w_{i}\right)-\{a\}\right) \cup\{b\}$ for some $a \in N\left(w_{i}\right)$ and some $b \notin N\left(w_{i}\right)$. But the set $N\left(w_{j}\right)$ contains exactly one of $a$, $\bar{a}$. Then $b$ must be $\bar{a}$. Therefore, a single exchange is precisely replacing a vertex by its buddy, which means that two vertices in $G(\beta)$ are adjacent if and only if one of them can be obtained from the other by replacing a vertex from $U$ by its buddy. Now since $U=\left\{u_{1}, \bar{u}_{1}, u_{2}, \bar{u}_{2}, \ldots, u_{m}, \bar{u}_{m}\right\}$, we can represent each vertex $N(w)$ of $G(\beta)$ by a binary string $r_{1} r_{2} \cdots r_{m}$, where for $i=1,2, \ldots, m$, we put

$$
r_{i}= \begin{cases}0, & u_{i} \in N(w), \\ 1, & \bar{u}_{i} \in N(w),\end{cases}
$$

thus two vertices of $G(\beta)$ are adjacent if and only if their binary representations differ at exactly one place. This is just the binary representation of the hypercube $Q_{m}$.

It is shown in [8] that $G(2 m, 2 m)$ has exactly $8 m$ automorphisms. This indicates that $G(2 m, 2 m)$ has a lot of symmetry. The next result reflects the higher symmetry $G\left(2 m, 2^{m}\right)$ possesses.

Theorem 4.7. For any integer $m \geq 3$, the automorphism group $\operatorname{Aut}\left(G\left(2 m, 2^{m}\right)\right)$ has order $2^{m} m !$.

Proof. Let $f$ be an automorphism of $G\left(2 m, 2^{m}\right)$. Then, since $f$ preserves degrees of vertices and $|U| \neq|W|$, it follows by Lemma 2.6 that the vertex $f\left(w_{1}\right)=w$ must belong to $W$. Then $f\left(N\left(w_{1}\right)\right)=N(w)$, and hence since $f$ preserves distances between vertices, we must have $f(u)=\overline{f(\bar{u})}$ for every vertex $u \in U-N\left(w_{1}\right)=N\left(\bar{w}_{1}\right)$. Now for any vertex $x \in W-\left\{w_{1}\right\}$, by the construction of $G\left(2 m, 2^{m}\right)$, there exists a unique vertex $y \in W$ such that $N(y)=f(N(x))$ and hence $f(x)=y$. This gives an automorphism $f$ of $G\left(2 m, 2^{m}\right)$. Thus an automorphism of $G\left(2 m, 2^{m}\right)$ is completely defined by specifying the image of every vertex in the closed neighborhood $N\left[w_{1}\right]$. From the proof above, the image of $w_{1}$ can be any vertex from $W$, and the set of images of the vertices from $N\left(w_{1}\right)$ must only be the set of neighbors of $f\left(w_{1}\right)$. This implies that we have exactly $2^{m}$ ways to choose $f\left(w_{1}\right)$ and then $m$ ! ways to choose the set of images of the vertices from $N\left(w_{1}\right)$. Therefore, $G\left(2 m, 2^{m}\right)$ has exactly $2^{m} m$ ! automorphisms.

One can easily verify that the $S$-graphs of diameter 4 and order at most 14 , already characterized in Corollary 4.5, are bipartite self complementary. The following result assures that this is the case for any $S$-graph of diameter 4 .

Theorem 4.8. If $G$ is an S-graph of diameter 4, then $G$ is bipartite self complementary.

Proof. Let $U=\left\{u_{1}, u_{2}, \ldots, u_{k}, \bar{u}_{1}, \bar{u}_{2}, \ldots, \bar{u}_{k}\right\}$ and $W=\left\{w_{1}, w_{2}, \ldots, w_{t}, \bar{w}_{1}, \bar{w}_{2}, \ldots, \bar{w}_{t}\right\}$ be the partite sets of $G$ which is a subgraph of $K_{2 k, 2 t}$. Then, by Lemma 2.6, each vertex $x$ from $U$ is adjacent to exactly half of the vertices of $W$, and $\bar{x}$ is adjacent to the other half. Thus, the oneto-one correspondence $\phi$ from $V(G)$ to $V\left(K_{2 k, 2 t}-E(G)\right)$, which fixes every vertex in $W$ and sends every vertex in $U$ to its buddy, preserves adjacency.

Consequently, both $G(2 m, 2 m)$ and $G\left(2 m, 2^{m}\right)$ are bipartite self complementary.

Finally, we close this section by the following result including some properties of $G(2 m, 2 m)$ and $G\left(2 m, 2^{m}\right)$ concerning girth, domination number, and being Eulerian.

Theorem 4.9. (i) The girth of each of the graphs $G(2 m, 2 m)$ and $G\left(2 m, 2^{m}\right)$, where $m \geq 3$, is 4; while the graph $G(4,4)$ has girth 8. 
(ii) The domination number of any S-graph of diameter 4 is 4 . Hence, each of $G(2 m, 2 m)$ and $G\left(2 m, 2^{m}\right)$ has domination number 4 .

(iii) Any S-graph of diameter 4 is Eulerian if and only if the cardinality of each of its partite sets is divisible by 4 . Hence, the graphs $G(2 m, 2 m)$ and $G\left(2 m, 2^{m}\right)$ are Eulerian if and only if $m$ is even.

Proof. (i) The graph $G(4,4)$ is the 8-cycle. Now consider $m \geq 3$. In the graph $G(2 m, 2 m)$, we have $N\left(u_{1}\right)=\left\{w_{1}, w_{2}, \ldots, w_{m}\right\}$ and $N\left(u_{2}\right)=\left\{w_{1}, w_{2}, \ldots, w_{m-1}\right\} \cup\left\{\bar{w}_{m}\right\}$. Then $u_{1} w_{1} u_{2} w_{2} u_{1}$ is a 4-cycle in the bipartite graph $G(2 m, 2 m)$ which implies that the girth of $G(2 m, 2 m)$ is 4 . Now since $G\left(2 m, 2^{m}\right)$ is bipartite, then by Theorem 4.1, its girth is also 4 .

(ii) Let $G$ be an $S$-graph of diameter 4 and partite sets $U$ and $W$. Then by Lemma 2.6, each vertex in one of the partite sets is adjacent to exactly half of the vertices of the other partite set. Thus, $G$ cannot have a dominating set with number of elements less than 4. But for any two vertices $u, w$, where $u \in U$ and $w \in W$, we have $N(u) \cup N(\bar{u})=W$ and $N(w) \cup N(\bar{w})=U$. Therefore, $\{u, \bar{u}, w, \bar{w}\}$ is a dominating set of $G$.

(iii) Let $G$ be an $S$-graph of diameter 4 and partite sets $U$ and $W$. By Lemma 2.6, each vertex of $G$ has degree either $(1 / 2)|U|$ or $(1 / 2)|W|$. Therefore, the degree of every vertex of $G$ is even if and only if both $|U|$ and $|W|$ are multiples of 4 .

One might put for further research the following question. For an $S$-graph of diameter 4 and partite sets $U$ and $W$, where $2 m=|U| \leq|W|$, a sharp lower bound and a sharp upper bound of $|W|$ are $2 m$ and $2^{m}$, respectively. If $m>3$, then for each integer $t$ with $m<t<2^{m-1}$, what about the existence of an $S$-graph of diameter 4 and partite sets $U$ and $W$, where $2 m=|U|$ and $|W|=2 t$ ?

\section{Acknowledgment}

The authors are grateful to the referees for their valuable suggestions.

\section{References}

[1] A. Berman and A. Kotzig, "Cross-cloning and antipodal graphs," Discrete Mathematics, vol. 69, no. 2, pp. 107-114, 1988.

[2] F. Göbel and H. J. Veldman, “Even graphs,” Journal of Graph Theory, vol. 10, no. 2, pp. 225-239, 1986.

[3] Z. Chen, "On k-pairable graphs," Discrete Mathematics, vol. 287, no. 1-3, pp. 11-15, 2004.

[4] H. M. Mulder, "n-cubes and median graphs," Journal of Graph Theory, vol. 4, no. 1, pp. 107-110, 1980.

[5] H. M. Mulder, The interval function of a graph, vol. 132 of Mathematical Centre Tracts, Mathematisch Centrum, Amsterdam, 1980.

[6] K. R. Parthasarathy and R. Nandakumar, "Unique eccentric point graphs," Discrete Mathematics, vol. 46, no. 1, pp. 69-74, 1983.

[7] G. Chartrand and P. Zhang, Introduction to Graph Theory, McGraw-Hill, New York, NY, USA, 2005.

[8] S. Al-Addasi, "Diametrical graphs," M.S. thesis, University of Jordan, Amman, Jordan, 1993.

[9] F. A. Cotton, Chemical Applications of Group Theory, Wiley-Interscience, New York, NY, USA, 3rd edition, 1990.

[10] O. Kido, K. Kamitsuji, M. Kurumada, et al., "Morphological alteration upon phase transition and effects of oxygen impurities of chromium nanoparticles," Journal of Crystal Growth, vol. 275, no. 1-2, pp. e1745-e1750, 2005.

[11] Z. Lu, Y. Tang, L. Chen, and Y. Li, "Shape-controlled synthesis and characterization of $\mathrm{BaZrO}_{3}$ microcrystals," Journal of Crystal Growth, vol. 266, no. 4, pp. 539-544, 2004.

[12] S. Al-Addasi and H. Al-Ezeh, "Characterizing symmetric diametrical graphs of order 12 and diameter 4," International Journal of Mathematics and Mathematical Sciences, vol. 30, no. 3, pp. 145-149, 2002. 
[13] V. Chepoi, "Basis graphs of even Delta-matroids," Journal of Combinatorial Theory. Series B, vol. 97, no. 2, pp. 175-192, 2007.

[14] K. T. Huber, J. H. Koolen, and V. Moulton, "On the structure of the tight-span of a totally splitdecomposable metric," European Journal of Combinatorics, vol. 27, no. 3, pp. 461-479, 2006.

[15] T. Gangopadhyay and S. P. R. Hebbare, "r-partite self-complementary graphs—diameters," Discrete Mathematics, vol. 32, no. 3, pp. 245-255, 1980.

[16] H.-J. Bandelt, H. M. Mulder, and E. Wilkeit, "Quasi-median graphs and algebras," Journal of Graph Theory, vol. 18, no. 7, pp. 681-703, 1994. 


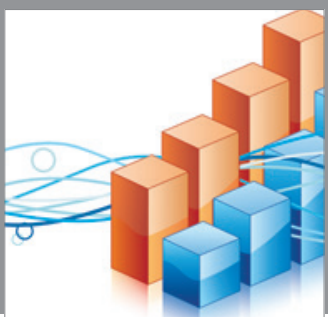

Advances in

Operations Research

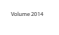

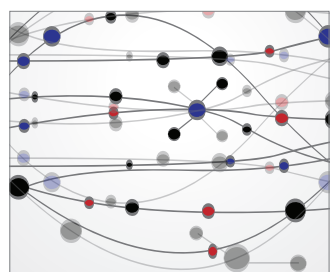

\section{The Scientific} World Journal
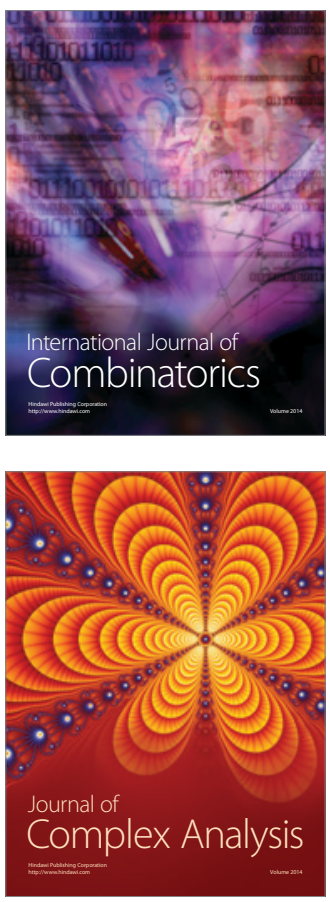

International Journal of

Mathematics and

Mathematical

Sciences
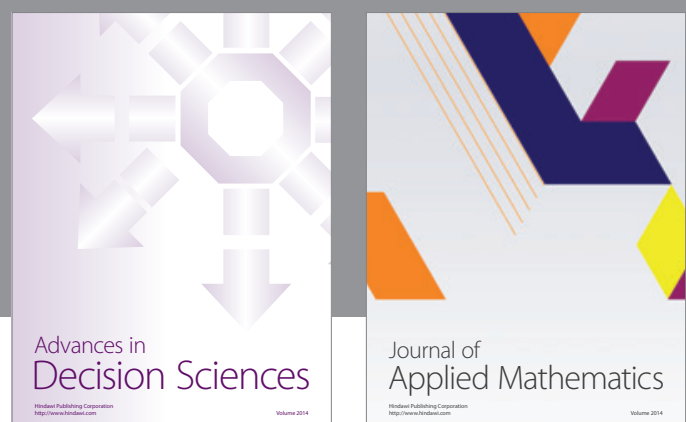

Journal of

Applied Mathematics
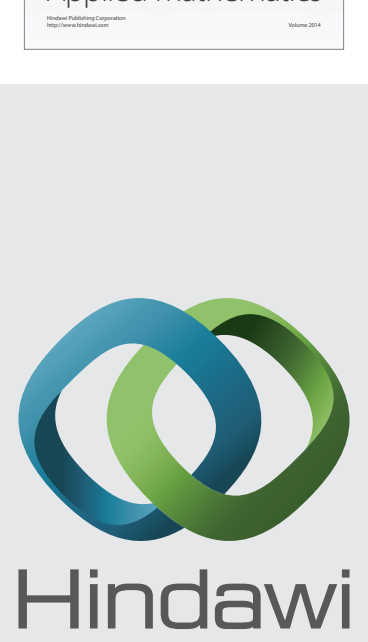

Submit your manuscripts at http://www.hindawi.com
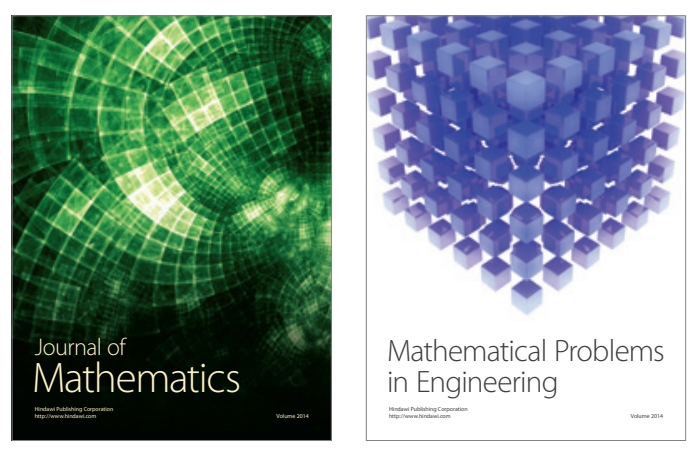

Mathematical Problems in Engineering
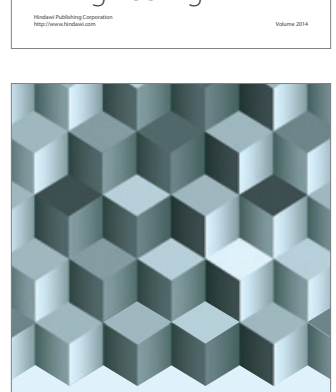

Journal of

Function Spaces
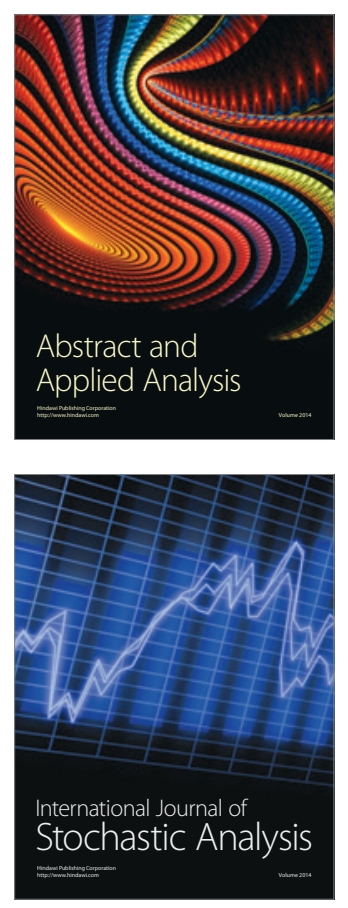

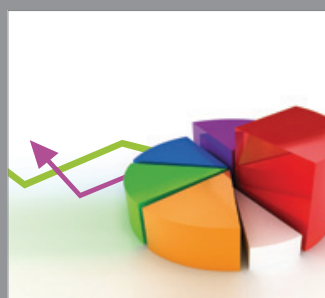

ournal of

Probability and Statistics

Promensencen
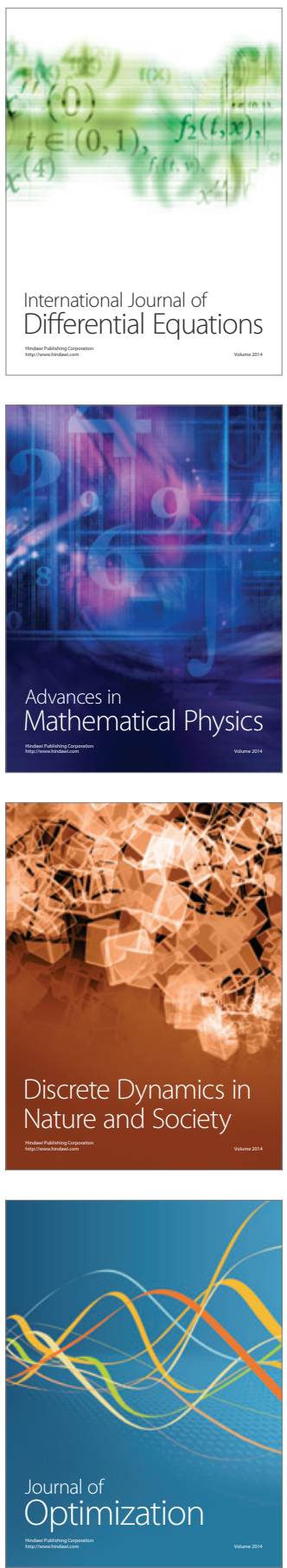